\title{
High linearity CMOS variable gain amplifier for UWB applications
}

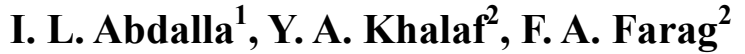 \\ ${ }^{1}$ Electronics and Communication, Egypt-Japan University for Science and Technology, Alexandria, Egypt \\ ${ }^{2}$ Electronics and Communication, Zagazig University, Zagazig, Egypt \\ Email address: \\ i.lotfi@msn.com (I. L. Abdalla), ykhalaf@vt.edu (Y. A. Khalaf), ffarag@zu.edu (F. A. Farag)
}

\section{To cite this article:}

I. L. Abdalla, Y. A. Khalaf, F. A. Farag. High Linearity CMOS Variable Gain Amplifier for UWB Applications. Science Journal of Circuits, Systems and Signal Processing. Vol. 1, No. 1, 2012, pp. 1-8. doi: 10.11648/j.cssp.20120101.11

\begin{abstract}
A large dynamic-range Programmable Variable Gain Amplifier (PVGA) suitable for Ultra Wide Band (UWB) applications is presented. The PVGA is composed of three programmable variable gain amplifier stages followed by an output buffer. Such wide bandwidth allows our proposed PVGA to be used in multi-standard protocols. Power reduction is developed for the variable gain amplifier stages. Thorough analyses of the mid-band gain and noise are presented; and design tradeoffs are carefully handled. The PVGA circuit is designed and simulated in $0.13 \mu \mathrm{m}$ IBM-CMOS process; the overall PVGA with buffer consumes $25 \mathrm{~mA}$ from a $1.5 \mathrm{~V}$ supply. The PVGA achieves $54.5 \mathrm{~dB}$ dynamic-range (DR), 17.6 $\mathrm{dBm}$ IIP3, -42.31 dB THD at peak-to-peak differential output voltage of $1 \mathrm{~V}$, and frequency $400 \mathrm{MHz}$ Moreover; the proposed circuit reports a good noise performance; the average integrated noise is $121.6 \mathrm{nV} / \sqrt{\mathrm{Hz}}$ at minimum gain of $-0.5 \mathrm{~dB}$.
\end{abstract}

Keywords: Automatic Gain Control (AGC), Variable Gain Amplifier (VGA), Programmable Variable Gain Amplifier (PVGA), Digitally-controlled Variable Gain Amplifier (DVGA), Buffer, CMOS Analog Integrated Circuits, Low Voltage, Wide Bandwidth

\section{Introduction}

The variable gain amplifier (VGA) is an indispensable building block to maximize the dynamic range of modern wireless communication systems [1], [2]. It is also widely used in medical equipment, hearing aids, disk drives, and so on [3]-[5]. A VGA is typically employed in a feedback loop to realize automatic gain control (AGC). The VGA of an AGC loop is used to control the transmission signal power or to adjust the received signal amplitude. There are two possible approaches to control the gain of the VGA. One is to build a discrete gain step VGA with a digital control signal [6], [7]; and the other is to design a VGA controlled by an analog gain- control signal [1]-[5]. Basically, digitally-controlled VGAs use binary weighted arrays of resistors or capacitors for gain variations whereas analogcontrolled VGAs adopt a variable transconductance or a variable resistance to control the gain. VGA circuits based on various technologies such as bipolar, BiCMOS, and CMOS have been introduced in the literature (e.g. [1]-[4]).

An important VGA requirement is to have a linear-indecibel gain control characteristic, where the gain of the VGA changes exponentially with the control signal. The exponential gain control is required to achieve a wide dynamic-range and to maintain the AGC loop settling-time independent of the input signal level [8].

Another important aspect of a wideband VGA is to attain a large bandwidth. There are many systems for high-speed data communications such as ultra-wideband (UWB) systems, wireless local area networks (LANs), and Bluetooth. These systems provide a high data rate with relatively low power consumption in short-range wireless communications. For high-speed data communication, the bandwidth of a VGA must be very wide. Therefore, a wideband VGA is a key component.

While the density of the devices in Nanoscale technology is continually increasing, the interconnect lines across a chip increase significantly. Such long interconnects mandate the requirement of driving large capacitances [12]-[15]. Moreover, in some situations, circuits should have large fan-out. For these reasons, buffers are frequently used in analog and mixed signal circuits. Well-designed buffers should drive large capacitive load with wide bandwidth, minimum power consumption, low distortion, and low loading effect.

In this paper, we propose a new highly-linear wideband 
programmable variable gain amplifier PVGA. Our PVGA parameters are compared to [16] - [19] to clarify the merits of the proposed circuit. It could obtain a high linearity, wide bandwidth, and large dynamic-range with large capacitive load and high swing.

Section II describes the architecture of the proposed wideband PVGA supported by VGA-cell analysis. The design of the main blocks in the PVGA is also explained. Noise and linearity performance of the PVGA are analyzed. The implementation and simulation results are presented in Section III. The results are also analyzed and discussed. Conclusions are given in section IV.

\section{Design of the Wideband CMOS PVGA}

The proposed programmable variable gain amplifier PVGA, as shown in Fig. 1, consists of a number of variable gain amplifier stages (cells) followed by a buffer to drive the load. A binary control-word is used to control the gain of the variable gain amplifiers. The bandwidth of such variable gain amplifier cell should be so wide that the overall bandwidth of the whole VGA design is achieved. In this section, we introduce a three-stage configuration to implement the PVGA with wide bandwidth, in which all VGAcells are identical. The Variable Gain Amplifier (VGA)cells implementation is considered and analyzed here. A novel VGA-cell, based on the Flipped Voltage Follower (FVF) [11] technique, is proposed with modifications to increase the headroom for the output swing and to enhance the transconductance of the VGA-cell. A new topology for the output buffer is also described and analyzed.

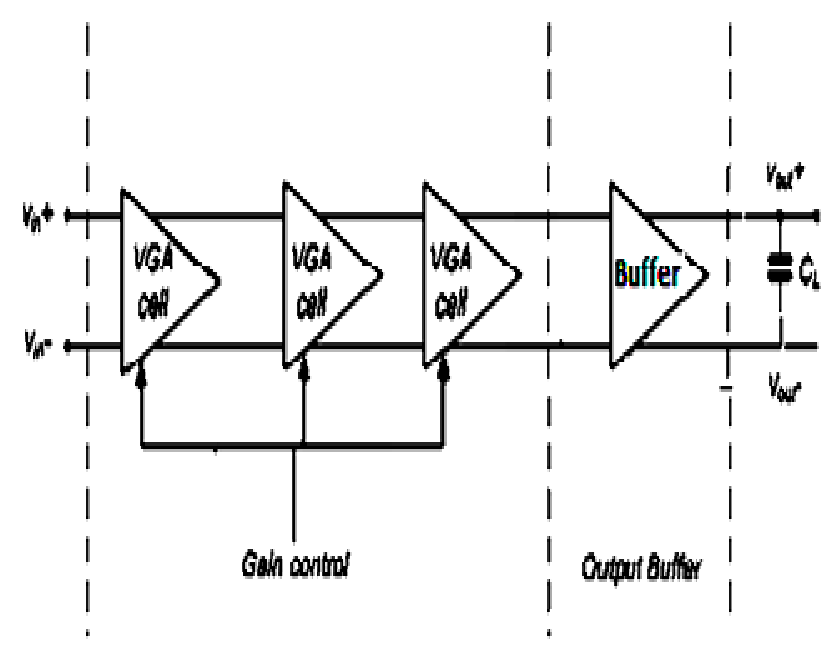

Fig. 1. Architecture of the overall PVGA.

\subsection{Design of VGA-Cell}

The complete circuit of the proposed VGA-cell is shown in Fig. 2. It demonstrates the signal-processing circuit, the common-mode-feedback circuit, and the bias circuit.

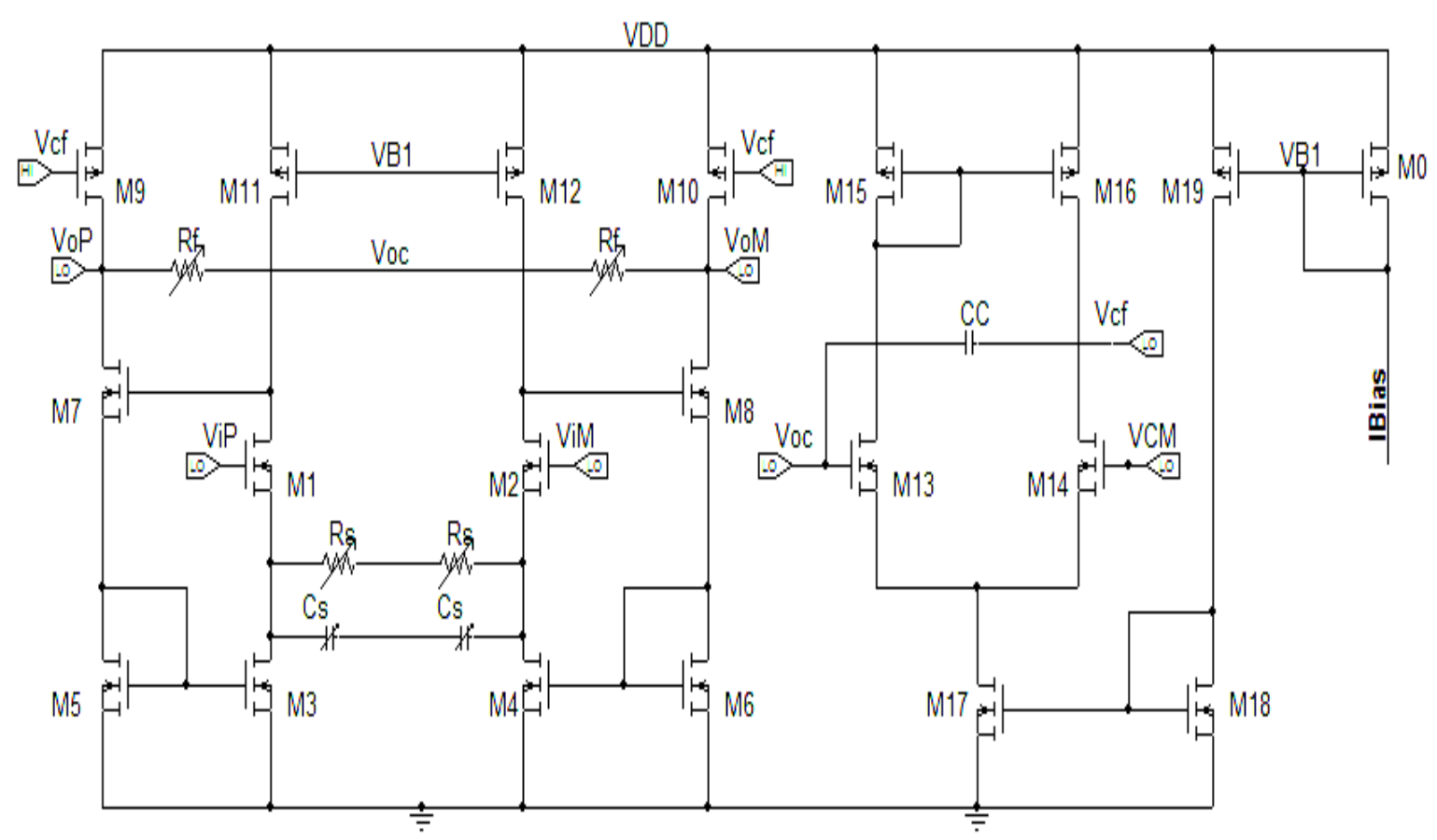

Fig. 2. Schematic diagram of the proposed VGA-cell.

The VGA-cell consists of a CMOS differential amplifier, in which the $R_{f}$ and $R_{s}$ are used to vary the gain. $C s$ is inserted to widen out the bandwidth. $\mathrm{M}_{1}$ and $\mathrm{M}_{2}$ are used to transfer the differential-voltage signal across $R_{s}$, which in turn is converted into differential-current flowing through $\mathrm{M}_{3}$ and $\mathrm{M}_{4}$. The differential signal current is mirrored to 
the output branches through $\mathrm{M}_{5}$ and $\mathrm{M}_{6} \cdot \mathrm{M}_{7}$ and $\mathrm{M}_{8}$ are connected in Wilson current-mirror like-connectivity to define drain voltage of $\mathrm{M}_{1}$ and $\mathrm{M}_{2}$; and to provide larger headroom for the swing at these points in contrast to [11]. It also provides large output resistance at the output nodes $\mathrm{V}_{\mathrm{OP}}$ and $\mathrm{V}_{\mathrm{OM}}$. This VGA-cell is typically used to get the coarse and fine steps by controlling $\mathrm{R}_{\mathrm{s}}$ and $\mathrm{R}_{\mathrm{f}}$, respectively.

The output voltage $\left(\mathrm{V}_{\mathrm{OP}}\right)$ half-circuit of the lowfrequency small-signal model for the differential amplifier is shown in Fig. 3. The output voltage can be written as,

$$
V_{o P}=-g_{m 5} V_{g s 3}\left(R_{f} / / r_{o 9}\right)
$$

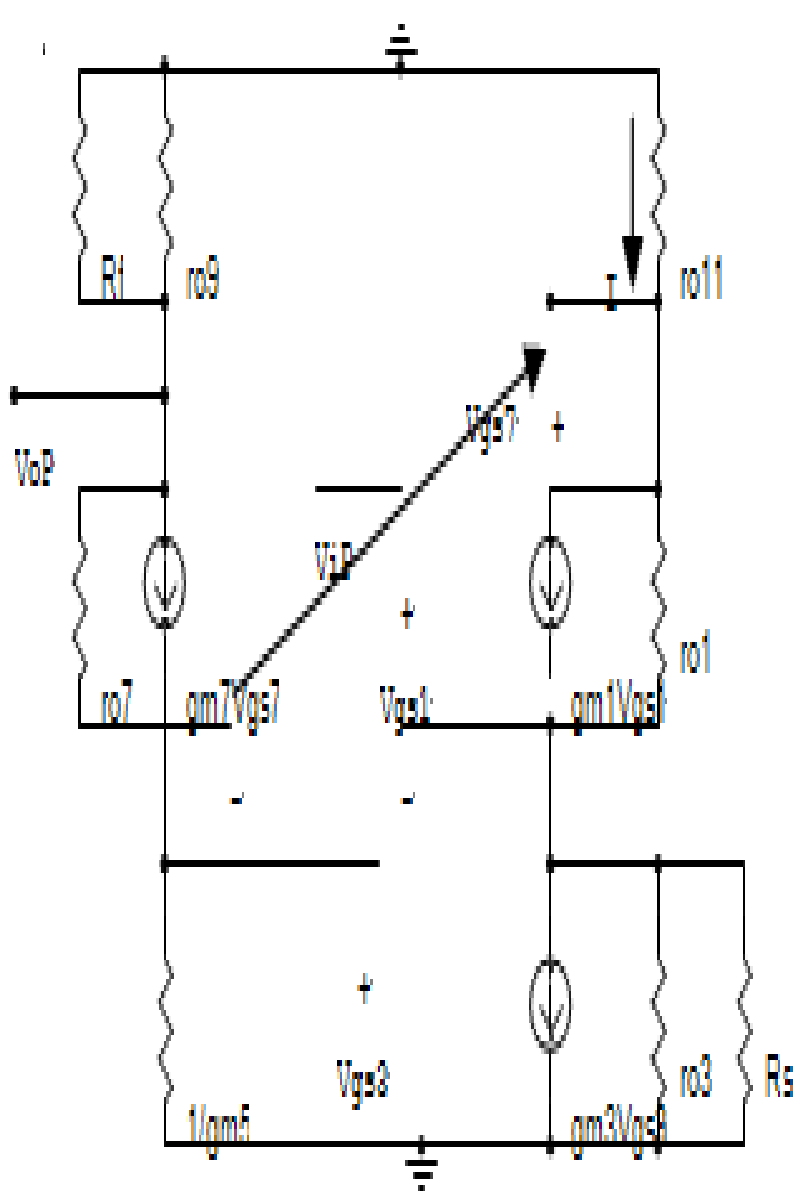

Fig. 3. Small-signal model of VGA circuit. (Half of the circuit is shown for clarity).

where $\mathrm{g}_{\mathrm{m} 5}$ is the transconductance of $\mathrm{M}_{5-6}, \mathrm{~V}_{\mathrm{gs} 3}$ is the voltage between gate and source of $\mathrm{M}_{3,4}$, and $\mathrm{r}_{09}$ is the output resistance of the transistor $\mathrm{M}_{9-10} . \mathrm{r}_{05}$ is ignored, compared to $1 / \mathrm{g}_{\mathrm{m} 5}$

The input voltage ViP of the differential amplifier is then,

$$
V_{i P}=V_{g s 1}+\left(I-g_{m 3} V_{g s 3}\right)\left(R_{s} / / r_{o 3}\right)
$$

where $\mathrm{I}$ is the current flowing into $\mathrm{r}_{\mathrm{o} 11}$.

$$
I=\frac{g_{m 1} r_{o 1} V_{g s 1}+g_{m 3}\left(R_{s} / / r_{o 3}\right) V_{g s 3}}{\left[\left(R_{s} / / r_{o 3}\right)+r_{o 1}+r_{o 11}\right]}
$$

Applying KVL on the output branch, $\mathrm{V}_{\mathrm{gs} 7}$ can be calculated as,

$$
V_{g s 7}=\frac{\left\lfloor 1+g_{m 5}\left(r_{o 7}+\left(R_{f} / / r_{o 9}\right)\right\rfloor V_{g s 3}\right.}{g_{m 7} r_{o 7}}
$$

On the other hand, $\mathrm{V}_{\mathrm{gs} 3}$ can be related to $\mathrm{V}_{\mathrm{gs} 7}$ as,

$$
V_{g s 3}+V_{g s 7}=-I r_{o 11}
$$

Using equations (1) through (5) and doing some mathematical manipulation, we can calculate the output voltage gain $A_{V}$,

$$
\begin{gathered}
A_{V} \equiv \frac{V_{o P}}{V_{i P}}=\frac{\left(R_{f} / / r_{o 9}\right)}{\left(R / / r_{o 3}\right)} \\
{\left[\frac{1}{\left(\frac{g_{m 3} / g_{m 5}}{1+\frac{\left(R_{s} / / r_{o 3}\right)}{r_{o 1}+r_{o 11}}}\right)+\left[\frac{X}{A}\right]\left(\frac{\left.1+\frac{g_{m 1} r_{o 1}\left(R_{s} / / r_{o 3}\right)}{\left[\left(R_{s} / / r_{o 3}\right)+r_{o 1}+r_{o 11}\right]}\right)}{g_{m 5}\left(R_{s} / / r_{o 3}\right)}\right)}\right]}
\end{gathered}
$$

$r_{011}$ represents the resistance of the current source. If $r_{011}$ is relatively large, the output voltage gain $A_{V}$ can be approximated as,

$$
A_{V} \equiv \frac{V_{o P}}{V_{i P}}=\frac{\left(R_{f} / / r_{o 9}\right)}{\left(R_{s} / / r_{o 3}\right)} \frac{g_{m 5}}{g_{m 3}}
$$

It is worthy to mention that the effective transconductance seen by the degeneration resistance $R_{s}$ is enhanced resulting in small voltage loss from the input to the source of $\mathrm{M}_{1}$. As seen from (7), the voltage gain $A_{V}$ is independent of transistor parameters except for $\mathrm{r}_{\mathrm{o} 9}$, and $\mathrm{r}_{\mathrm{o} 3}$. In reality, $\mathrm{R}_{\mathrm{f}}$ and $R_{s}$ are chosen much smaller than $r_{09}$, and $r_{03}$, respectively.

$A_{V}$ can then be approximately expressed as,

$$
A_{V} \cong \frac{R_{f}}{R_{S}} n
$$

where $\mathrm{n}$ is the ratio $\left(\mathrm{g}_{\mathrm{m} 5} / \mathrm{g}_{\mathrm{m} 3}\right)$. As a result, the gain of the circuit is stable across process corner variations. In our design, $n$ is chosen to be equal to unity whereas the ratio $R_{f}$ $/ \mathrm{R}_{\mathrm{s}}$ should be designed to implement linear-in-dB gain.

\subsection{Noise Analysis}

Half of the differential amplifier with equivalent noise sources is shown in Fig. 4. 


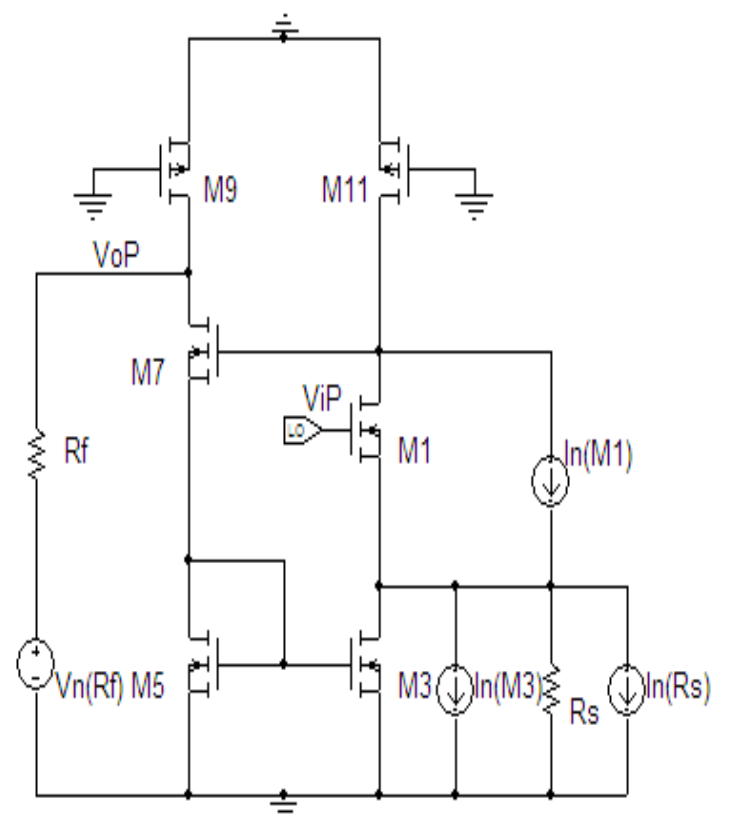

Fig. 4. Calculation of the input-referred noise of the proposed VGA-cell.

The drain-source noise current generators of MOSFETs are considered. For noise sources of $\mathrm{M}_{3,4}$ and the resistance $\mathrm{R}_{\mathrm{s}}$, the total output noise can be calculated as,

$$
\overline{v_{n, \text { out }}^{2}}=\frac{g_{m 7}^{2}\left(R_{f} / / r_{o 9}\right)^{2} a^{2}}{\left(1+\frac{a g_{m 3} g_{m 7}}{g_{m 5}}\right)^{2}} i_{n}^{2}
$$

where,

$$
a=\left(\frac{g_{m 5^{r} o 11}}{g_{m 5}+g_{m 7}}\right)\left(\frac{g_{m 1} R_{s}}{1+g_{m 1} R_{s}}\right) \text { and, } \bar{i}_{n}^{2}=\overline{i_{n, M 3}^{2}}+\overline{i_{n, R_{s}}^{2}}
$$

gmi and roi are the transconductance and output resistance of the transistor Mi.

Referring to the input port,

$$
\overline{v_{\mathrm{n}, \text { in }}^{2}}=\frac{\overline{v_{n, \text { out }}^{2}}}{\left|A_{V o}\right|^{2}}
$$

The input referred noise due to $M_{3,4}$ and $R_{s}$ can be approximately expressed as:

$$
\overline{v_{n, \text { in }}^{2}}=\left(\frac{1}{1+\frac{g_{m 7}}{g_{m 5}}}\right)^{2}\left(\frac{g_{m 1} R_{S}}{1+g_{m 1} R_{S}}\right)^{2} R_{S}^{2} \overline{i_{n}^{2}}
$$

The noise due to input transistor $M_{1}$ and resistance $R_{f}$ can be added to estimate the total input-referred noise as,

$$
\overline{v_{n, \text { in (tot })}^{2}}=\frac{1}{g_{m 1}^{2}} i_{n 1}^{2}+\left(\overline{v_{n, R_{f}}^{2}}+\overline{v_{n, \text { out }}^{2}}\right)\left|\frac{1}{A_{V 0}}\right|^{2}
$$

where,

$$
\overline{i_{n, M_{i}}^{2}}=4 k T\left(\frac{2}{3} g_{m i}\right) \Delta f+K \frac{I_{D i}^{a}}{f} \Delta f, \overline{i_{n, R_{s}}^{2}}=\frac{4 k T}{R_{S}} \Delta f
$$

And

$$
\overline{v_{n, R_{f}}^{2}}=4 k T R_{f} \Delta f
$$

At low-gain settings, $\mathrm{A}_{\mathrm{v} 0}$ becomes small and so the last term in (11), defined by (10), dominates the noise performance of the circuit. As a result, $\mathrm{R}_{\mathrm{S}}$ should be chosen small enough for good noise performance. In contrast, at largegain settings, noise performance is dominated by the first term in (11) (input transistors $\mathrm{M}_{1}$ and $\mathrm{M}_{2}$ ) and consequently $\mathrm{g}_{\mathrm{m} 1}$ should be maximized.

\subsection{Output Buffer}

Our proposed buffer circuit [10] is shown in Fig. 5. The circuit has achieved large transconductance without increasing the aspect ratio of the main differential transistors $\mathrm{M}_{1}$ and $\mathrm{M}_{2}$. The basic idea of the buffer is to improve the bandwidth based on the Flipped Voltage Follower (FVF) by enhancing the transconductance of $\mathrm{M}_{3}\left(\mathrm{M}_{4}\right)$ through the feedback loop $\mathrm{M}_{1}, \mathrm{M}_{7}$, and $\mathrm{M}_{5}\left(\mathrm{M}_{2}, \mathrm{M}_{8}\right.$, and $\left.\mathrm{M}_{6}\right)$.

The circuit is biased by two pairs of current sources $\left(\mathrm{M}_{9}\right.$ and $\left.\mathrm{M}_{10}\right)$ and $\left(\mathrm{M}_{11}\right.$ and $\left.\mathrm{M}_{12}\right)$; the former pair supplies low current to the main differential transistors, and so small loading-effect is observed at the input of the buffer. The latter pair is used to enhance the required current needed for driving large capacitive load. The feedback is accomplished through the level shifter $\mathrm{M}_{5}$ and $\mathrm{M}_{7}\left(\mathrm{M}_{6}\right.$ and $\left.\mathrm{M}_{8}\right)$ to increase the swing headroom for $\mathrm{M}_{1}\left(\mathrm{M}_{2}\right)$, and consequently the linearity.

The output voltage (VoP) half-circuit of the small-signal model is shown in Fig. 6. One half of the circuit is only considered for simplicity without loss of generality.

A similar analysis, to what has been done in Section II.A, can be conducted to calculate the output voltage gain $\mathrm{AV}$,

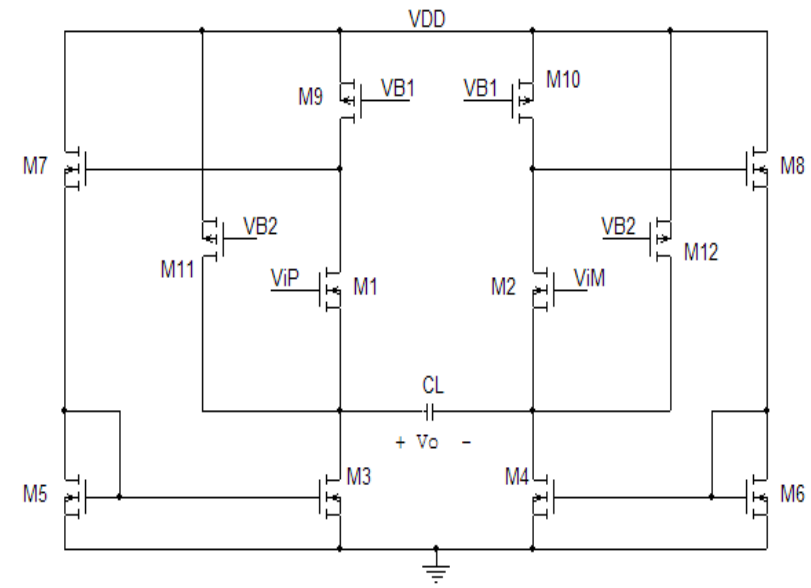

Fig. 5. Proposed buffer circuit [10]. 


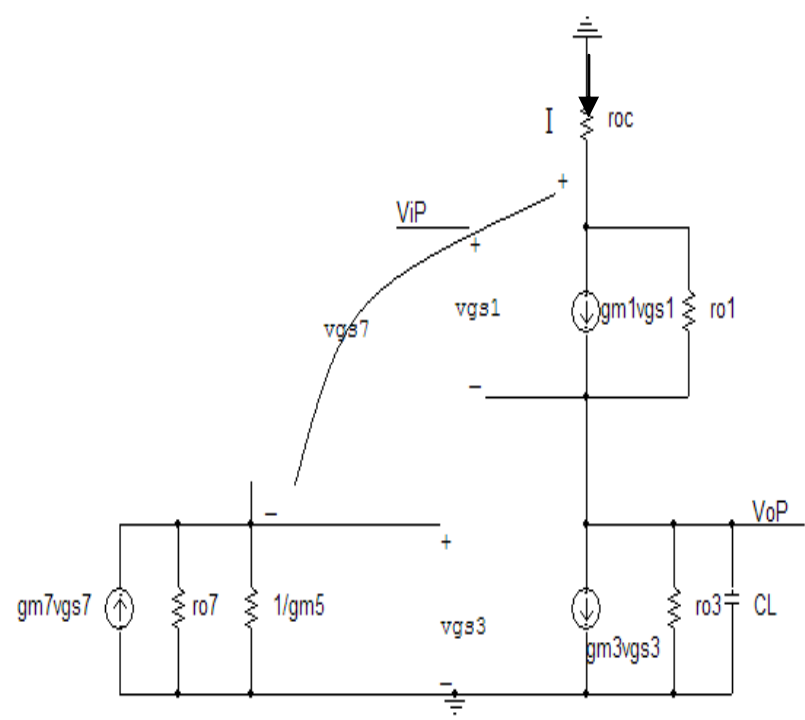

Fig. 6. Small-signal model of the proposed buffer. (half of the circuit is shown for clarity).

$$
A_{v}=\frac{A_{O}}{1+j \omega \frac{C_{L}}{g_{\text {meff }}} A_{O}}
$$

Where $A_{o}$ is the DC gain defined by,

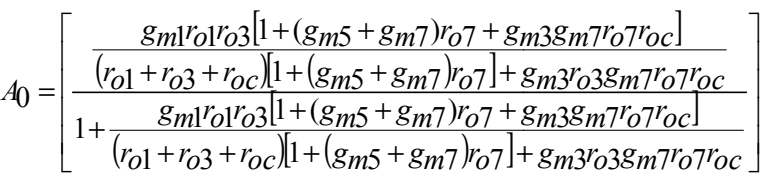

and

$g_{m e f f}=\left[\frac{g_{m 1} r_{o 1} r_{o 3}\left[1+\left(g_{m 5}+g_{m 7}\right) r_{o 7}+g_{m 3} g_{m 7} r_{o 7} r_{o c}\right]}{r_{o 3}\left(r_{o 1}+r_{o c}\right)\left[1+\left(g_{m 5}+g_{m 7}\right) r_{o 7}\right]}\right]$

If $r_{\mathrm{oc}} \mathrm{r}_{\mathrm{o} 1}$ and $\mathrm{r}_{\mathrm{o} 7}$ are very large and the term $\mathrm{r}_{\mathrm{o} 3} \mathrm{~g}_{\mathrm{m} 3}$ is much greater than unity, the effective transconductance can be reduced to,

$$
g_{m e f f}=g_{m 1}\left[1+\frac{g_{m 3} g_{m 7} r_{O C}}{\left(g_{m 5}+g_{m 7}\right)}\right]
$$

From (14), the overall transconductance is approximately improved by the closed loop gain from input to the ac current of $\mathrm{M}_{3}-\mathrm{M}_{4}$, through $\left(\mathrm{M}_{1}, \mathrm{M}_{7}, \mathrm{M}_{5}\right.$, and $\left.\mathrm{M}_{3}\right)$, which is very large.

The overall transconductance was improved by a factor proportional to approximately $\mathrm{g}_{\mathrm{m}} \mathrm{r}_{\mathrm{oc}}$ without increasing the basic transistor size. On the other hand, the THD is superior for our proposed cell compared to the FVF cell.

\section{PVGA Implementation and Simula- tion Results}

\subsection{Variable Gain Amplifier}

The PVGA is implemented in $0.13-\mu \mathrm{m}$ IBM CMOS process. The design equations, derived in the previous section, are used to guide our design. The PVGA achieves the required bandwidth of $400 \mathrm{MHz}$ with gain varying from $0.5 \mathrm{~dB}$ to $54 \mathrm{~dB}$ using 5-bits control-word. It consists of three identical VGA-cells. Rs is used to generate coarsegain steps of $16 \mathrm{~dB}$ each whereas $\mathrm{Rf}$ is used to generate fine-gain steps of $2 \mathrm{~dB}$ each. Compensation capacitor array Cs has been also used in parallel with Rs to introduce a programmable zero into the transfer function that can be employed to widen out the bandwidth at large gain settings. The overall simulated variable gain amplifier current dissipation is $21 \mathrm{~mA}$.

Fig. 7 shows the gain plot versus gain-control word for the simulated and analytical results equation (8); the gain is clearly linear-in-dB. There is also good agreement between analytical and simulated plots across wide gain dynamic range from $-0.5 \mathrm{~dB}$ up to $54 \mathrm{~dB}$. The insensitivity to process variation has been also studied, where there are only $0.5 \mathrm{~dB}$ deviations from the results of the typical mean process parameters.

Fig. 8 shows the frequency response for different gain settings. The bandwidth decreases as the gain increases, however bandwidth could be kept at its minimum value of $400 \mathrm{MHz}$ by controlling the zero location without significant increase in power consumption. While the bandwidth at the maximum gain can achieve $400 \mathrm{MHz}$, the bandwidth at the minimum gain extends to $2 \mathrm{GHz}$. It is also worthy to mention that the peak occurs outside the bandwidth of interest.

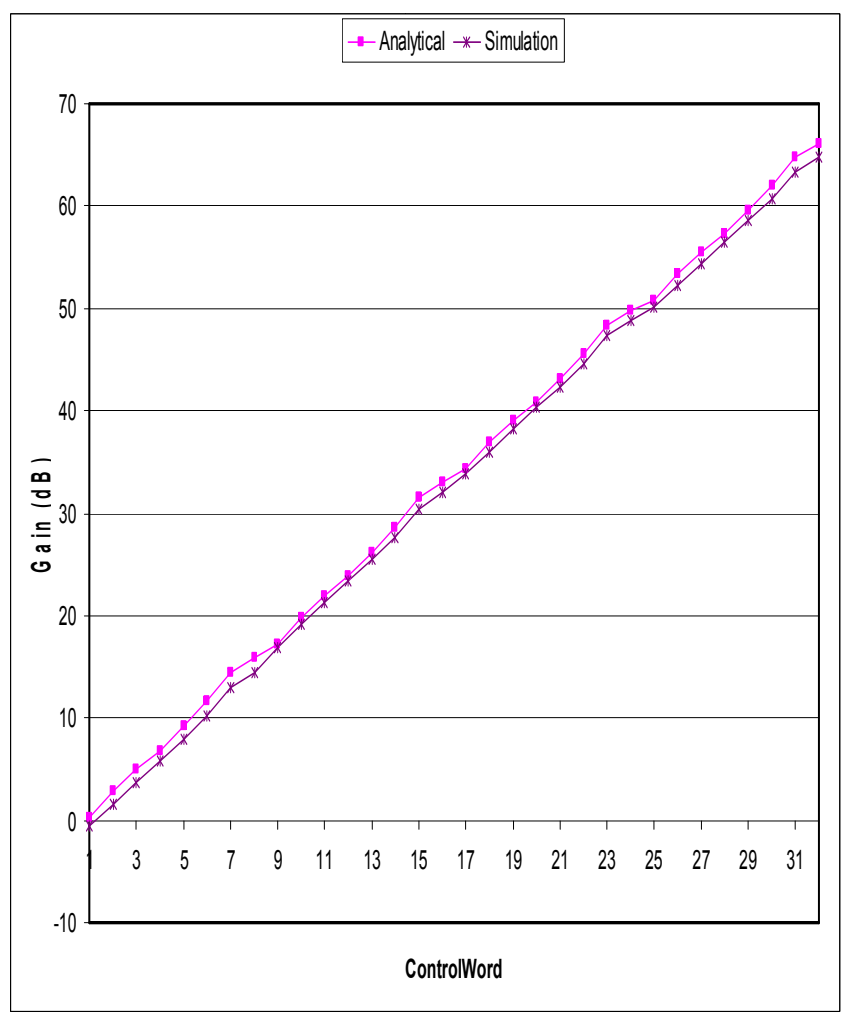

Fig. 7. Linear-in-dB gain versus control word. 


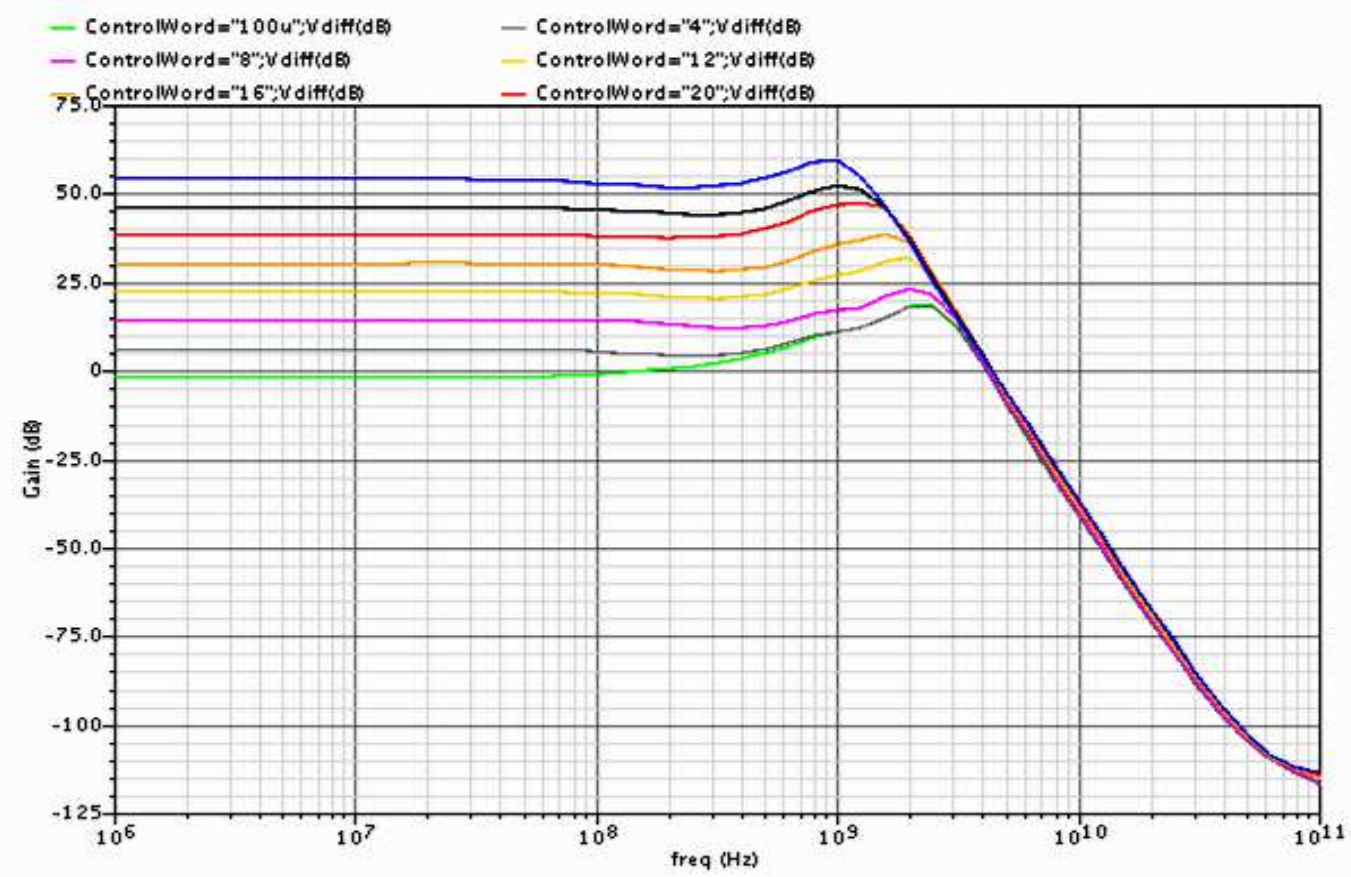

Fig. 8. Gain versus frequency for different gain settings.

The linearity performance of VGA is characterized by two-tone IP3 test. It was measured by applying two tones of $100 \mathrm{MHz}$ and $120 \mathrm{MHz}$ at the lowest gain mode. The result is shown in Fig. 9 where IIP3 is equal to $17.6 \mathrm{dBm}$.

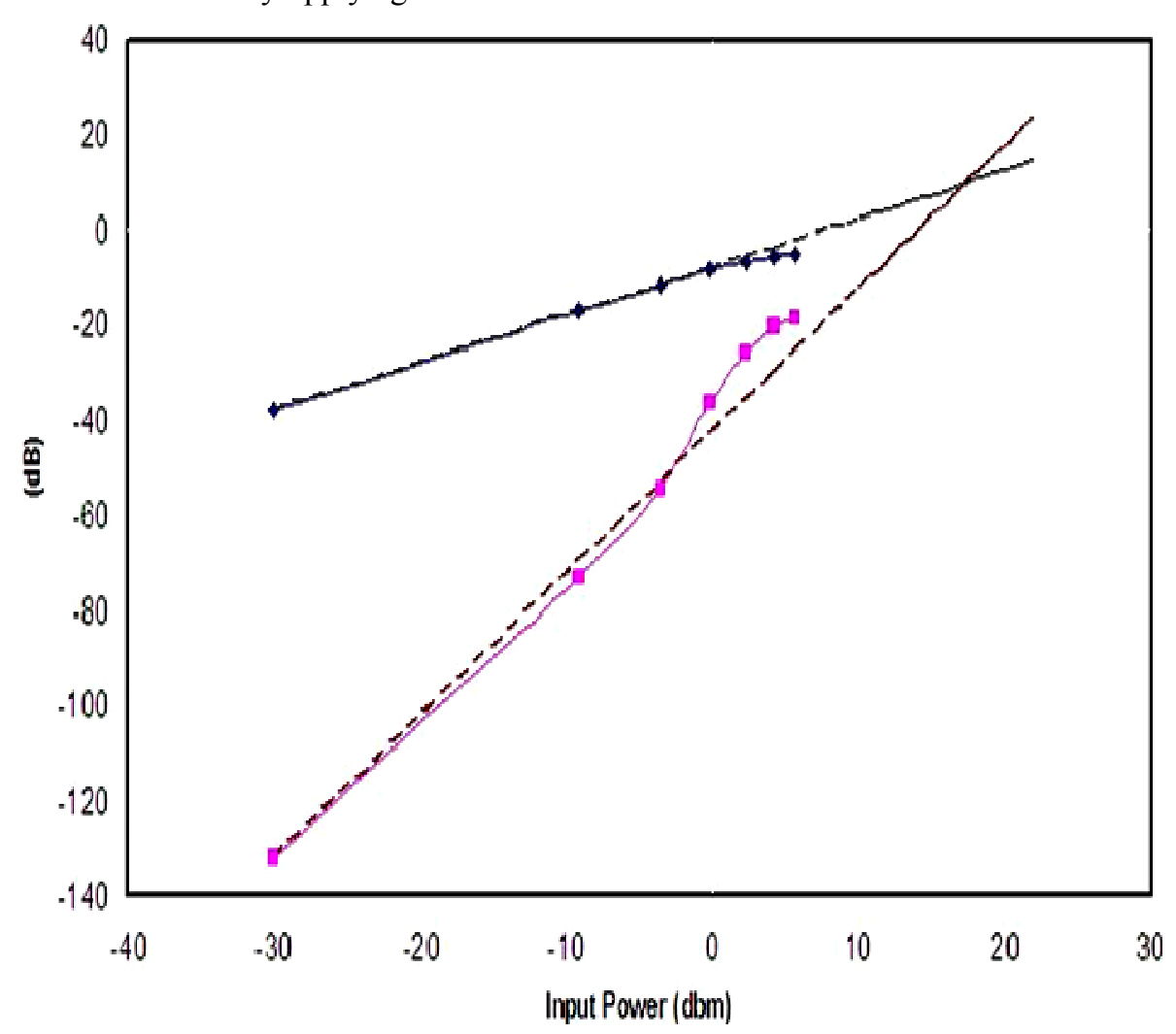

Fig. 9. The IIP3 at the lowest gain mode.

Table 1 compares our work against others' work in the literature. The GBW/power is taken as a figure of merit for a reasonable comparison. Our PVGA shows the highest figure-of-merit. The circuit also shows reasonable noise per- 
formance which can be certainly improved at the expense of higher power consumption.

Table 1. Performance summary and comparison.

\begin{tabular}{|c|c|c|c|c|c|}
\hline Parameters & [16] & [17] & [18] & [19] & This Work \\
\hline Technology & $0.13 \mu \mathrm{m}$ & $0.18 \mu \mathrm{m}$ & $0.18 \mu \mathrm{m}$ & $0.18 \mu \mathrm{m}$ & $0.13 \mu \mathrm{m}$ \\
\hline Supply voltage & $1.5 \mathrm{~V}$ & $1.8 \mathrm{~V}$ & $1.8 \mathrm{~V}$ & $1.8 \mathrm{~V}$ & $1.5 \mathrm{~V}$ \\
\hline Bandwidth (MHz) & $183-548$ & 450 & 650 & $400 @ 54 \mathrm{~dB}$ & 1500 \\
\hline Gain range & $-5-65 \mathrm{~dB}$ & $3-47 \mathrm{~dB}$ & $0.7-60.7 \mathrm{~dB}$ & $-30-65 \mathrm{~dB}$ & $-0.5-54 \mathrm{~dB}$ \\
\hline IIP3 & & $20 \mathrm{dBm}$ & & & $17.6 \mathrm{dBm}$ \\
\hline Current dissipation & $13.4 \mathrm{~mA}$ & $15 \mathrm{~mA}$ & $8.9 \mathrm{~mA}$ & $12.2 \mathrm{~mA}$ & $21 \mathrm{~mA}$ \\
\hline $\mathrm{P}_{\text {diss }}(\mathrm{mW})$ & 20.1 & 27 & 16.02 & 21.96 & 31.5 \\
\hline THD @ freq=400MHz \& $V_{\text {od(p-p) }}=1 \mathrm{~V}$ & & & & & $\begin{array}{l}-49.47 \mathrm{~dB} @ 54 \mathrm{~dB} \\
-43.26 \mathrm{~dB} @-0.5 \mathrm{~dB}\end{array}$ \\
\hline Input referred noise & & & & & $121.6 \mathrm{nV} / \sqrt{\mathrm{Hz}} @-0.5 \mathrm{~dB}$ \\
\hline GBW/Power(MHz/mW) & 48438 & 7518 & 48507 & 53083 & 56103 \\
\hline
\end{tabular}

\subsection{Overall PVGA}

Table 2. Summary of the simulation results of the overall PVGA.

\begin{tabular}{lll}
\hline Parameters & $@-\mathbf{- 0 . 5} \mathbf{~ d B}$ & $@ \mathbf{5 4} \mathbf{~ d B}$ \\
\hline Bandwidth $(\mathrm{GHz})$ & 3.5 & 1.5 \\
Average Integrated Noise $(\mathrm{nV} / \sqrt{ } \mathrm{Hz})$ & 121.6 & 17.4 \\
THD (dB) @ 400 MHz and Vodp-p=1 & -40.04 & -42.31 \\
V. & 37.5 & \\
Power dissipation (mw) & \\
\hline
\end{tabular}

Table 2 summarizes the crucial simulation results of the overall programmable variable gain amplifier with the out- put buffer at maximum and minimum gain settings. The buffer is loaded with a capacitive single-ended load of $2 \mathrm{pF}$. The noise performance of the circuit is dominated by the VGA, the buffer noise is insignificant because of its large transconductances and so small input referred noise.

Fig. 10 shows the frequency response of the overall PVGA at the extreme gain settings. As seen from Fig. 10, the frequency response extends for more than $400 \mathrm{MHz}$. Although the small-signal bandwidth can be as large as 1.5 $\mathrm{GHz}$, the effective bandwidth is limited to $400 \mathrm{MHz}$ only, for output voltage swing of $1 \mathrm{Vdpp}$ with the aforementioned loading, owing to the slew-rate limitation.

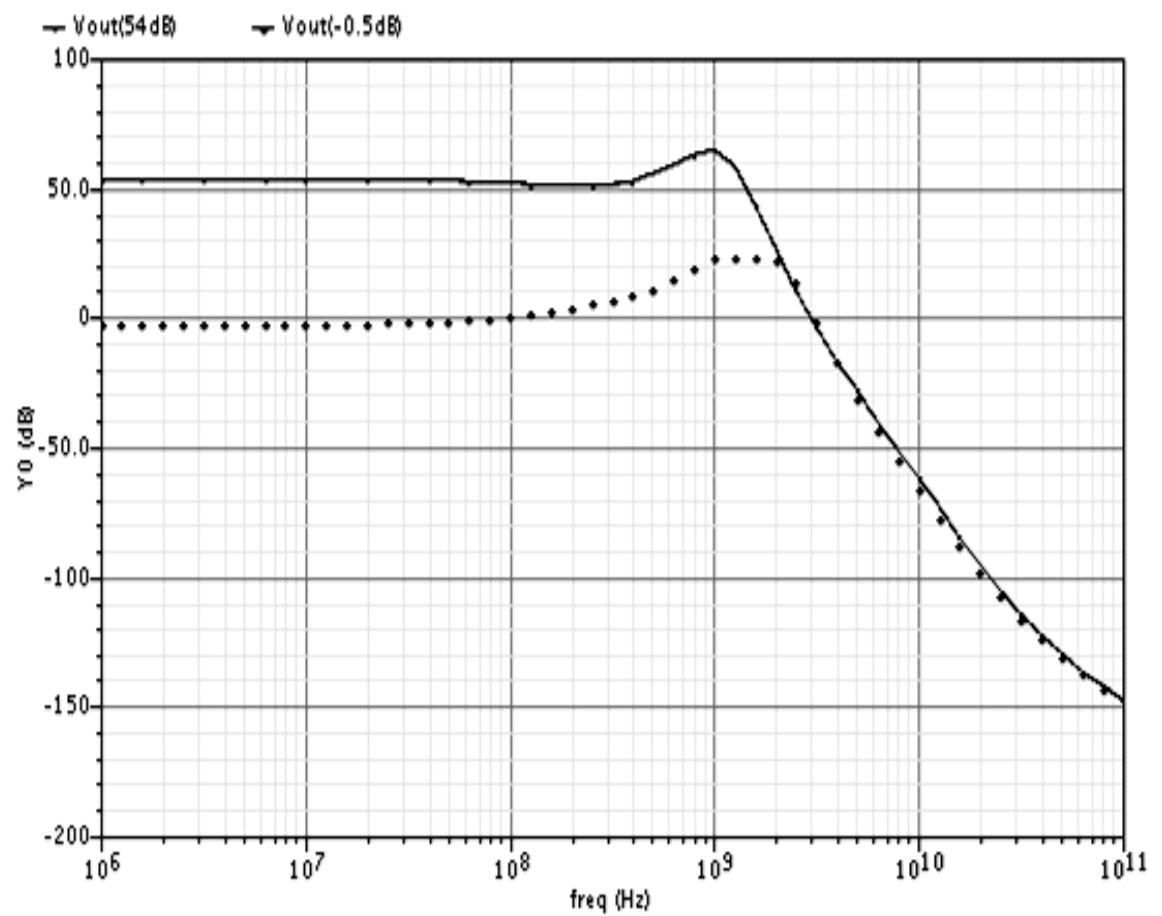

Fig. 10. Frequency response for the overall PVGA. 


\section{Conclusion}

This paper presents a low-voltage CMOS PVGA. It achieved wide bandwidth over wide dynamic gain range from $-0.5 \mathrm{~dB}$ to $54 \mathrm{~dB}$. With this circuit topology, the gain and bandwidth can be programmed independently. The circuit has been designed and implemented using $0.13 \mu \mathrm{m}$ IBM-CMOS process with supply voltage of $1.5 \mathrm{~V}$ and power dissipation of $37.5 \mathrm{~mW}$ to drive $2 \mathrm{pF}$ single-ended capacitive loads.

This PVGA can be applied to various applications, such as high performance industrial systems and baseband circuits in multi-standard wireline and wireless communications with wide bandwidth.

\section{Acknowledgements}

The authors gratefully acknowledge the technical support of the MOSIS Company for their support and provision of the design kit used in this work.

\section{References}

[1] J. K. Kwon, K. D. Kim, W. C. Song, and G. H. Cho, "Wideband high dynamic range CMOS variable gain amplifier for low voltage and low power wireless applications," Electron. Lett., vol. 39, no. 10, pp. 759-760, Mar. 2003.

[2] T. Yamaji, N. Kanou, and T. Itakura, “A temperature-stable CMOS variable-gain amplifier with $80-\mathrm{dB}$ linearly controlled gain range," IEEE J. Solid-State Circuits, vol. 37, no. 5, pp. 553-558, May 2002.

[3] W. M. Christopher, "A variable gain CMOS amplifier with exponential gain control," in VLSI Circuits Tech. Dig. Symp., pp. 146-149, Jun. 2000.

[4] P. Huang, L. Y. Chiou, and C. K.Wang, "A 3.3-V CMOS wideband exponential control variable-gain-amplifier," in Proc. IEEE Int. Circuits Syst. Symp., pp. I-285-I-288, May 1998.

[5] M. M. Green and S. Joshi, "A 1.5 V CMOS VGA based on pseudodifferential structures," in Proc. IEEE Int. Circuits Syst. Symp., pp. IV-461-IV-464, May 2000.

[6] P. Orsatti, F. Piazza, and Q. Huang, "A 71-MHz CMOS IFbaseband strip for GSM," IEEE J. Solid-State Circuits, vol. 35, no. 1, pp. 104-108, Jan. 2000.

[7] H. O. Elwan and M. Ismail, "Digitally programmable decibel-linear CMOS VGA for low-power mixed-signal applica- tions," IEEE Trans. Circuits Syst. II, Analog Digit. Signal Process., vol. 47, no. 5, pp. 388-398, May 2000.

[8] J. M. Khoury, "On the design of constant settling time AGC circuit," IEEE Trans. Circuits Syst. II, Analog Digit. Signal Process., vol. 45, no. 3, pp. 283-294, Mar. 1998.

[9] I. L. Abdel-Hafez, Yaser A. Khalaf, and F. A. Farag, "CMOS buffer amplifier for wide bandwidth applications, " IEEE International Conference on Design and Technology of Integrated Systems in NanoScale Era (DTIS'7), pp. 56-59, September 2007.

[10] Ramón González Carvajal, Jaime RamírezAngulo, ,Antonio J. López-Martín, Antonio Torralba,, Juan Antonio Gómez Galán,Alfonso Carlosena, , and Fernando Muñoz Chavero," The flipped voltage follower: a useful cell for low-voltage low-power circuit design," IEEE transactions on circuits and systems I: vol. 52, no. 7, July 2005.

[11] J. J. F. Rijns, "CMOS low-distortion high-frequency variable-gain amplifier," IEEE journal of solid-st/,te circuits, vol. 31, no. 7, July 1996.

[12] Kornika Moolpho and Jitkasame Ngarmnil, "Low voltage high-performance class-ab fgmos buffer," Circuits and Systems, APCCAS 2006. IEEE Asia Pacific Conference, pp. 1779 - 1782, Dec. 2006.

[13] Chih-Wen Lu and Yen-Chung Huang, "1.5 V large-driving class-AB buffer amplifier with quiescent current control," Electronics Letters 8th, Vol. 40 No. 1, Jan. 2004

[14] S. Baswa, A.J. Lo'pez-Martı'n, R.G. Carvajal, and J. Ramı'rez-Angulo, "Low-voltage power-efficient adaptive biasing for CMOS amplifiers and buffers," Electronics Letters 19th, Vol. 40 No. 4, Feb. 2004.

[15] Kh. Hadidi, J. Sobhi, A. Hasankhaan, D. Muramatsu, and T. Matsumoto, "A novel highly linear CMOS buffer," Electronics, Circuits and Systems, 1998 IEEE International Conference on Volume 3, 7-10, PP. 369 - 371 vol.3 , Sept. 1998.

[16] Seunghyun Jang, Seung-Sik Lee, Sang-Sung Choi, and Kwang-Chun Lee,"A CMOS Baseband Receiver for Wireless Broadband Communications," Feb. 7-10, 2010 ICACT 2010.

[17] Yen Ju The, Choong Foo Chung, and M. Annamalai Arasu, "A 3-47dB gain, $+20 \mathrm{dBm}$ IIP3, $400 \mathrm{MHz}$ VGA for a pulsebased UWB in $0.18 \mu \mathrm{m}$ CMOS," ISIC 2009.

[18] J. Cheng, F.Y. Huang*, L.H. Wu, Y. Tian, and N. Jiang, “A High-linearity, 60-dB Variable Gain Amplifier with Dual DC-offset Cancellation for UWB Systems," Sept. 4, WICOM.2009.

[19] Chan Tat Fu and Luong, H., "A CMOS linear-in-dB highlinearity variable-gain amplifier for UWB receivers," SolidState Circuits Conference, 2007. ASSCC '07. 